\section{No long-term benefit of prednisolone plus azathioprine in membranous nephropathy}

Patients with idiopathic membranous nephropathy are sometimes treated with a combination of the antiproliferative immunosuppressant azathioprine and the corticosteroid prednisolone; however, results of a retrospective analysis of data from the Sheffield Kidney Institute in the UK indicate that this dual regimen has no long-term clinical benefits. In membranous nephropathy patients with poor prognosis, the authors recommend that other therapies, such as cyclophosphamide, ciclosporin or chlorambucil, be used.

Of the 50 patients with nephrotic syndrome secondary to biopsy-proven idiopathic membranous nephropathy, 33 received oral prednisolone (initial dose $0.5-1.0 \mathrm{mg} / \mathrm{kg} /$ day; tapered to $5-10 \mathrm{mg} /$ day over 12 months) plus azathioprine (initial dose $2 \mathrm{mg} / \mathrm{kg} /$ day; tapered to $50 \mathrm{mg} /$ day over 12 months). The mean treatment period was 26 months. The remaining patients $(n=17)$ received no immunosuppressive drugs. There were no significant biochemical or histological differences between the two groups at baseline.

After 10 years, levels of serum creatinine had doubled in $40 \%$ of subjects, and end-stage renal failure had developed in $20 \%$. Doubling of baseline serum creatinine, end-stage renal failure and remission of proteinuria occurred in a similar number of patients in the treated and untreated groups. In patients who did not develop end-stage renal failure, mean levels of serum creatinine, serum albumin, urinary protein and blood pressure did not differ between those who were treated and those who were not treated. Adverse effects, such as squamous cell carcinoma, pneumococcal septicemia and leukopenia, occurred in $27 \%$ of patients receiving immunosuppressive therapy.

Original article Goumenos DS et al. (2006) Prednisolone and azathioprine in membranous nephropathy: a 10-year follow-up study. Clin Nephrol 65: 317-323

\section{Dialysis fluid is at risk of organic contamination}

Although patients on hemodialysis are exposed to hundreds of liters of dialysis fluid each week, there are no guidelines regarding acceptable concentrations of organic contaminants in this fluid; the most commonly used values are those recommended for potable water. The organic compound trichloroethylene is a potentially carcinogenic environmental pollutant that is associated with liver damage and neuropathy. Researchers in Italy have found that trichloroethylene can accumulate in components of hemodialysis water treatment systems.

The maximum concentration of trichloroethylene allowed in drinking water by the Italian Health Ministry is $10 \mu \mathrm{g} / \mathrm{l}$, but routine monitoring at one dialysis center found concentrations of $60 \mu \mathrm{g} / \mathrm{l}$ in reverse osmosis water, in addition to high concentrations in various dialysis system devices. Patients $(n=15)$ were transferred to other centers until trichloroethylene levels in the dialysis water had been normalized, following replacement of the activated charcoal, ionic-exchange resins, microfilters and polyvinyl chloride pipes. No patients showed any clinical sign of trichloroethylene intoxication, either at the time contamination was detected or when the center was reopened 1 month later. Nevertheless, extrapolation of elimination curves indicated that initial blood trichloroethylene levels were about $1.48 \mu \mathrm{g} / \mathrm{l}$, approximately 15 times higher than those in the unexposed population. Follow-up of seven patients for 30-60 days after reopening of the center revealed that trichloroethylene levels decreased over time, reaching concentrations similar to those in the unexposed population after 60 days.

Dialysis water should be monitored regularly for organic substances such as trichloroethylene that have a high affinity for the blood compartment.

Original article Poli D et al. (2006) Organic contamination in dialysis water: trichloroethylene as a model compound. Nephrol Dial Transplant 21: 1618-1625

\section{Effect of complement 3 polymorphisms on long-term outcomes in renal transplant recipients}

In humans, the immune response effector complement 3 exists as two main allotypes: complement 3 fast (C3F) and complement 3 slow (C3S). A C3F allele in donor kidneys might confer a long-term graft survival benefit to recipients who lack this variant. This is the exciting 